\title{
Fe de errores de «Hipercolesterolemia familiar: artículo de revisión»
}

\section{Familial hypercholesterolemia: review article}

Alonso Merchán ${ }^{a}$, Álvaro J. Ruiz ${ }^{b}$, Rafael Campo $^{c}$, Carlos E. Prada $^{d}$, Juan M. Toro ${ }^{\mathrm{e}}$, Robinson Sánchez ${ }^{\mathrm{c}}$, Juan E. Gómez ${ }^{\mathrm{f}}$, Nicolás I. Jaramillo ${ }^{\mathrm{g}}$, Dora I. Molina ${ }^{\mathrm{h}}$, Hernando Vargas-Uricoechea ${ }^{i}$, Sherien Sixto ${ }^{j}$, Javier M. Castro ${ }^{k}$, Adalberto E. Quintero', Mauricio Coll ${ }^{m}$, Stase Slotkus ${ }^{n}$, Andrés Ramírez ${ }^{\circ}$, Harry Pachajoa ${ }^{\circ}$, Fernando A. Ávila ${ }^{p}$ y Rodrigo Alonso ${ }^{\mathrm{q}}$

\footnotetext{
a Educación Continuada Fundación Clínica Shaio, Bogotá, Colombia

b Pontificia Universidad Javeriana, Bogotá, Colombia

c Fundación Cardiovascular de Colombia, Bucaramanga, Colombia

' Centro de Medicina Genómica y Metabolismo, Fundación Cardiovascular de Colombia, Bucaramanga, Colombia

e Hospital Pablo Tobón Uribe. Universidad de Antioquia, Medellín, Colombia

${ }^{f}$ Fundación Valle de Lili, Cali, Colombia

s Universidad CES. Laboratorio cardiovascular, Clínica Las Américas, Medellín, Colombia

h Clinical Research Association, Manizales, Colombia

' Departamento de Medicina Interna. Universidad del Cauca, Popayán, Colombia

j Servicio de rehabilitación cardiaca. Instituto del Corazón de Bucaramanga, Sede Bogotá, Bogotá, Colombia

k Cardiología pediátrica. Clínica de Falla Cardiaca y Trasplante Cardiaco Pediátrico. Fundación Cardiovascular de Colombia, Bucaramanga, Colombia

' Cardiodiagnóstico SA. Centro de investigaciones Fundación del Caribe para la Investigación Biomédica, Barranquilla, Colombia

m Universidad Nacional de Colombia. Centro Médico Almirante Colón, Bogotá, Colombia

${ }^{\mathrm{n}}$ Fundación Clínica Shaio, Bogotá, Colombia

- Centro de Investigaciones en Anomalías Congénitas y Enfermedades Raras (CIACER). Facultad de Ciencias de la Salud. Universidad Icesi, Cali, Colombia

p Facultad de Ciencias de la Salud, Universidad Icesi, Cali, Colombia

q Clínica Las Condes, Santiago de Chile
}

Respecto al artículo «Hipercolesterolemia familiar: artículo de revisión» Rev Colomb Cardiol. 2016;23 Supl 4:4-26. El editor del presente documento ofrece disculpas debido a un error involuntario que se evidencia en el concepto emitido en la página 15, párrafo, segundo que hace alusión al evolocumab donde dice «... pero con la dosificación mensual se observa mayor variabilidad en los niveles de CLDL debido al consumo de los ACm que tienden a presentarse al final de las cuatro semanas», y precisa que esta información no está vigente ni corresponde a la fuente citada.

DOI: 10.1016/j.rccar.2016.05.002

Véase contenido relacionado en DOI: http://dx.doi.org/10.1016/j.rccar.2016.05.002 